\title{
Amino acid permease 3 (aap3) coding sequence as a target for Leishmania identification and diagnosis of leishmaniases using high resolution melting analysis
}

\author{
Karl Erik Müller ${ }^{1 *+}$ (D), Ricardo Andrade Zampieri ${ }^{2 \dagger}$, Juliana Ide Aoki ${ }^{2}$, Sandra Marcia Muxel², \\ Audun Helge Nerland ${ }^{1}$ and Lucile Maria Floeter-Winter ${ }^{2}$
}

\begin{abstract}
Background: The leishmaniases comprise a spectrum of clinical manifestations caused by different species of Leishmania. Identification of species is important for diagnosis, treatment and follow-up management. However, there is no gold standard for species identification. High resolution melting analysis (HRM) offers a possibility to differentiate Leishmania species without the need for processing of the PCR-product. The amino acid permease 3 (aap3) gene is an exclusive target for trypanosomatids and is conserved among Leishmania spp., thus it can be a valuable target for an HRM assay for diagnosis of the leishmaniases.

Results: The HRM dissociation profiles of three amplicons targeting the aap3-coding region allowed the discrimination of L. (Leishmania) donovani, L. (L.) infantum, L. (L.) major, L. (L.) tropica, L. (L.) mexicana, L. (L.) amazonensis, L. (Viannia) braziliensis, L. (V.) guyanensis, L. (V.) lainsoni, L. (V.) naiffi and L. (V.) shawi using DNA from promastigote cultures. The protocol was validated with DNA samples from clinical infection in humans and a cat, naturally infected sand flies, and experimentally infected mice.

Conclusions: HRM analysis using the aap3 coding sequence as target is a relatively cheap, fast and robust strategy to detect and discriminate Leishmania species from all the endemic regions worldwide. The target and method proved to be useful in clinical, field and experimental samples, thus it could be used as a tool in diagnosis as well as ecological and epidemiological studies.
\end{abstract}

Keywords: PCR, HRM, Infectious diseases, Leishmania discrimination

\section{Background}

The leishmaniases are a group of diseases caused by Leishmania spp. Clinical presentations range from self-healing cutaneous lesions to potentially lethal visceral leishmaniasis [1]. It is defined by the World Health Organization (WHO) as a neglected tropical disease, meaning it is underreported, underestimated, underfunded and underprioritized by the

\footnotetext{
*Correspondence: karl.muller@uib.no

${ }^{\dagger}$ Karl Erik Müller and Ricardo Andrade Zampieri contributed equally to this work.

'Department of Clinical Science, Faculty of Medicine, University of Bergen, Postboks 7804, 5020 Bergen, Norway

Full list of author information is available at the end of the article
}

pharmaceutical industry and often by public health authorities alike [2]. Over 350 million people are at risk of being infected, and it is estimated that 20,000 to 40,000 die each year of the leishmaniases [3]. In some regions, it is considered as largely an anthroponotic disease, while in others it is a zoonosis. The reservoir of the parasite can be domestic, sylvatic, and in some areas, both. Over 20 different species of Leishmania can cause the disease in humans $[1,4]$. The species that cause disease in humans are grouped into two subgenera: Leishmania (Leishmania) and Leishmania (Viannia), based on biological features of the parasites [5]. Species identification is important for clinical diagnosis,

(c) The Author(s). 2018 Open Access This article is distributed under the terms of the Creative Commons Attribution 4.0 International License (http://creativecommons.org/licenses/by/4.0/), which permits unrestricted use, distribution, and 
prognosis, treatment and follow-up management. For example, some L. (Viannia) species, such as $L$. (V.) braziliensis, are known to cause cutaneous lesions and may later reappear as mucocutaneous lesions, thus requiring systemic treatment, while other lesions infected by strains known to only cause cutaneous manifestations may be treated with local treatment or observation therapy [6].

The leishmaniases can be diagnosed in various ways, all with their strengths and limitations. However, there is no gold standard for diagnosing the diseases. Microscopy is useful in a high-endemic setting, but lacks sensitivity and needs a trained microscopist, not easily found in most non-endemic areas. Diagnostic tests involving serology, such as the direct agglutination test (DAT) and lateral flow immunochromatographic tests can also be useful. Especially lateral flow immunochromatographic tests can give a rapid diagnosis, are simple to use, easy to interpret and relatively cheap - all qualities which are very important in a low-resource setting in many endemic areas. However, serological tests may vary greatly in their sensitivity and specificity between endemic regions [7]. Furthermore, serology is not able to determine the species causing the disease. Diagnosis involving nucleic acid detection is valuable due the high sensitivity and specificity, and for the potential ability to quantify and identify the infecting species. There is a plethora of possible techniques and an equal amount of possible targets [8].

Real-time PCR, followed by high-resolution melting analysis (HRM), generates thermodynamic differences in the dissociation profile of amplicons resulting in specific signatures of polymorphisms due to small differences in nucleotide composition [9]. HRM is rapid, comparatively little laborious and a relatively cheap method where the post-PCR treatment is contained in the tubes with small risk for lab-contamination. HRM has been used for identification of other infectious agents [10-13]. In earlier work, we have already shown that HRM can be a valuable tool for Leishmania genotyping, using hsp70 as a target [14]. AAP3 is an amino acid transporter, which mediates uptake of lysine, histidine, phenylalanine, citrulline and arginine, with the highest affinity for the last one [15-20]. AAP3 is involved in the polyamine pathway, essential for parasite replication [17, 21-23]. The coding sequence for AAP3 is conserved among Leishmania spp., indicating its value as the chosen target [17]. We already demonstrated aap3 as an attractive target for detecting Leishmania, by a real-time PCR method, but this approach did not discriminate the species [24]. In this paper, we describe the use of the aap3 coding sequence as target for differentiation of Leishmania spp., using HRM analysis. The aap3-HRM method showed to be a specific and sensitive tool to differentiate Leishmania spp., using reference strain cultures and validated using clinical samples, naturally infected sand flies and experimentally infected mice samples.

\section{Methods}

\section{Organisms}

Promastigotes of Leishmania spp. (see Table 1) were grown at $25{ }^{\circ} \mathrm{C}$ in M199 medium containing Earle's salts, supplemented with $10 \%$ fetal bovine serum, $40 \mathrm{mM}$ HEPES (pH 7.4), $100 \mu \mathrm{M}$ adenine, $5 \mathrm{mg} / \mathrm{l}$ hemin, 0.05 $\mathrm{mg} / \mathrm{ml}$ streptomycin and $4550 \mathrm{U} / \mathrm{ml}$ penicillin. Trypanosoma cruzi (Y-strain), Crithidia fasciculata (TCC-039) and Endotrypanum schaudinni (MCHO/BR/80/M6159) were grown at $28{ }^{\circ} \mathrm{C}$ in liver-tryptose medium supplemented with $10 \%$ fetal bovine serum and $0.05 \mathrm{mg} / \mathrm{ml}$ streptomycin and $4550 \mathrm{U} / \mathrm{ml}$ penicillin. Trypanosoma brucei (Lister 427) was grown at $28{ }^{\circ} \mathrm{C}$ in SDM-79 medium (LGC Biotecnologia, Cotia, São Paulo, Brazil), supplemented with $10 \%$ fetal bovine serum, $0.05 \mathrm{mg} / \mathrm{ml}$ streptomycin and $4550 \mathrm{U} / \mathrm{ml}$ penicillin. Mammalian DNA from BALB/c mouse and Wistar rat were obtained from the DNA repository of the Laboratory of Trypanosomatidae at Physiology - IB-USP and were used as negative controls (see Additional file 1: Figure S1 and Additional file 2: Figure S2).

\section{Naturally and experimentally infected samples}

To validate the standardized protocols, samples previously identified by other diagnosis tests for the leishmaniases were used as a template in aap3-HRM assays [14, 26]. Human paraffin-embedded samples (from patients from Hospital das Clínicas da Universidade de São Paulo or Hospital da Irmandade da Santa Casa de Misericórdia de São Paulo), a sample from an infected cat (from Instituto de Medicina Tropical de São Paulo - USP), naturally infected sand flies (from Superintendência de Controle de Endemias de São Paulo), and samples from experimentally infected mice (from Instituto de Biociências USP) were included.

\section{DNA extraction}

The Leishmania strains used as assay-standards, T. cruzi, T. brucei, C. fasciculata, E. schaudinni and the mammalian DNA was isolated using a modified salting out technique, as previously described [14]. The DNA from the additional strains used for specificity studies was isolated using the QIAamp DNA Blood Mini Kit (Qiagen, Hilden, Germany), according to the manufacturer's instructions. DNA from mouse was isolated from whole blood and DNA from rat was isolated from liver-tissue, both by using the DNeasy Blood and Tissue Kit (Qiagen), according to the manufacturer's instructions. DNA quality and concentration were determined by NanoDrop ND-1000 Spectrophotometer (Thermo Scientific, USA). All DNA was stored at $-20{ }^{\circ} \mathrm{C}$ until further use.

For the paraffin-embedded tissues, a first step of washing was performed with xylol heated to $95{ }^{\circ} \mathrm{C}$ to remove paraffin, followed by repetitive washes of absolute ethanol. 
Table 1 Leishmania reference strains and additional strains used in this study with information about international code number, clinical form and host according to the World Health Organization classification [25]

\begin{tabular}{|c|c|c|c|c|}
\hline Strain & International code number & Clinical form & Isolated from & WHO reference strain \\
\hline \multicolumn{5}{|l|}{ Assay standard strains } \\
\hline L. (L.) donovani & MHOM/IN/80/DD8 & Visceral & Homo sapiens & Yes \\
\hline L. (L.) infantum & MCER/BR/1981/M6445 & Visceral & Cerdocyon thous & No \\
\hline L. (L.) tropica & MHOM/SU/60/OD & Cutaneous & Homo sapiens & No \\
\hline L. (L.) major & MHOM/IL/81/Friedlin & Cutaneous & Homo sapiens & No \\
\hline L. (L.) amazonensis & MHOM/BR/1973/M2269 & Cutaneous & Homo sapiens & Yes \\
\hline L. (L.) mexicana & MNYC/BZ/62/M379 & Cutaneous & Nyctomys sumichrasti & Yes \\
\hline L. (V.) lainsoni & MHOM/BR/81/M6426 & Cutaneous & Homo sapiens & Yes \\
\hline L. $(V$.$) braziliensis$ & MHOM/BR/1975/M2903 & Cutaneous & Homo sapiens & Yes \\
\hline L. (V.) guyanensis & MHOM/BR/1975/M4147 & Cutaneous & Homo sapiens & Yes \\
\hline L. $(V$.$) naiffi$ & MDAS/BR/1979/M5533 & na & Dasypus novemcinctus & Yes \\
\hline L. $(V$.$) shawi$ & MCEB/BR/84/M8408 & Cutaneous & Cebus apella & Yes \\
\hline \multicolumn{5}{|c|}{ Additional strains used for specificity studies } \\
\hline L. (L.) donovani & MHOM/CY/2006/CH33 & Visceral & Homo sapiens & No \\
\hline L. (L.) tropica & MHOM/SU/74/K27 & Cutaneous & Homo sapiens & Yes \\
\hline L. (L.) tropica & MHOM/MA/2000/INHW10 & Cutaneous & Homo sapiens & No \\
\hline L. (L.) major & MHOM/AF/2006/LEM5344 & Cutaneous & Homo sapiens & No \\
\hline L. (L.) major & MHOM/MA/2004/LEM4905 & Cutaneous & Homo sapiens & No \\
\hline L. (L.) major & MHOM/TN/2006/LPN296 & Cutaneous & Homo sapiens & No \\
\hline L. (L.) major & MRHO/SU/59/LV39 & na & Rhombomys opimus & No \\
\hline L. (L.) mexicana & MHOM/BZ/82/BEL21 & Cutaneous & Homo sapiens & Yes \\
\hline L. (L.) mexicana & MHOM/MX/93/CRE47 & Cutaneous & Homo sapiens & No \\
\hline L. (L.) mexicana & MHOM/MX/96/NAN01 & Cutaneous & Homo sapiens & No \\
\hline L. $(V$.$) braziliensis$ & MHOM/BR/75/M2904 & Cutaneous & Homo sapiens & Yes \\
\hline L. $(V$.$) braziliensis$ & MHOM/BR/87/LTB12MAR87 & Mucocutaneous & Homo sapiens & No \\
\hline L. (V.) guyanensis & MHOM/GF/94/22319 & Cutaneous & Homo sapiens & No \\
\hline L. (V.) naiffi & MHOM/GF/97/CRE88 & Cutaneous & Homo sapiens & No \\
\hline
\end{tabular}

Abbreviation: na not applicable

Then DNA was purified by an organic extraction with phenol-chloroform according Uliana et al. [27]. For sand flies, cultured Leishmania and mice samples, DNA was purified by silica columns, using DNeasy Blood \& Tissue Kit (Qiagen).

\section{Primer design}

The primers (listed in the Table 2) were designed based on the following sequences: L. (L.) amazonensis (HQ912026.1 and HQ912027.1), L. (V.) braziliensis (XM_001567050.2), L. (L.) major (XM_001685021.1), L. (L.) donovani

Table 2 Primer sequences used in this study based on the amino acid permease 3 (aap3) coding sequence, for amplicon 1 (Amp1), amplicon 2 (Amp2) and amplicon 3 (Amp3)

\begin{tabular}{|c|c|c|c|c|}
\hline Name & Sequence $\left(5^{\prime}-3^{\prime}\right)$ & Length (bp) & Orientation & Amplicon size $(b p)$ \\
\hline AAP3-Amp1-F & ATCCGCTACGTCTCCGCCATCGG & 23 & Forward & 123 \\
\hline AAP3-Amp1-R & CGTGGTGAAGTACTTCATGTCGC & 23 & Reverse & \\
\hline AAP3-Amp2-F & GCCGTCGATAAACACCCGAGC & 21 & Forward & 131 \\
\hline AAP3-Amp2-R & AAGCGGAAGATGATGTTGCGCCC & 23 & Reverse & \\
\hline AAP3-Amp3-F & GGCGGTCGCCTACATCAGCG & 20 & Forward & 140 \\
\hline AAP3-Amp3-R & CGGGCACCATGAACACGAGCCATA & 24 & Reverse & \\
\hline
\end{tabular}


(AY247004.1), L. (L.) infantum (XM_001467313.2) from GenBank, and $L$. (L.) aethiopica (LAEL147_000015800), $L$. (L.) mexicana (L.mxM.30.0870), L. (L.) tropica (LTRL590_ $310015200)$ and $L .(V$.) panamensis (LPAL13_000030300) from the TriTryp Database [28]. To predict melting temperatures (Tm), Oligo Calculator version 3.27 was used [29]. The nucleotide sequences of the amplicons and primer localizations are detailed in Additional file 3. A global BLAST search was performed for the three amplicon regions using the available amplicon sequences from the strains listed above.

\section{PCR assays}

Conventional PCR with the primers (Table 2) was performed for testing amplicon specificity. PCR reactions were performed using TopTaq Master Mix (Qiagen, Hilden, Germany) in a final volume of $25 \mu \mathrm{l}$ with $200 \mathrm{nM}$ of each primer and $25 \mathrm{ng}$ of genomic DNA as a template. The cycling conditions were as follows: initial denaturation at $94{ }^{\circ} \mathrm{C}$ for $3 \mathrm{~min}$, followed by 25 cycles of denaturation at $94{ }^{\circ} \mathrm{C}$ for $1 \mathrm{~min}$, annealing at $60{ }^{\circ} \mathrm{C}$ for $1 \mathrm{~min}$ and extension at $72{ }^{\circ} \mathrm{C}$ for $30 \mathrm{~s}$ and a final extension at $72{ }^{\circ} \mathrm{C}$ for $10 \mathrm{~min}$.

Real-time PCR was performed using MeltDoctor HRM Master Mix (Applied Biosystems, Foster City, CA, USA), according to the manufacturer's instructions. Final reaction volume was $20 \mu \mathrm{l}$, including $200 \mathrm{nM}$ of each primer and $25 \mathrm{ng}$ of genomic DNA as a template. Real time amplification conditions were as follows: an initial denaturation step at $95{ }^{\circ} \mathrm{C}$ for $5 \mathrm{~min}$, followed by 40 cycles of denaturation at $94{ }^{\circ} \mathrm{C}$ for $30 \mathrm{~s}$ and annealing/extension at $60{ }^{\circ} \mathrm{C}$ for $1 \mathrm{~min}$, with the acquisition of fluorescent signals at the end of each extension step, followed by the dissociation curve for HRM analysis. All reactions were performed in a Thermocycler PikoReal96 (Thermo Fisher Scientific, Walthan, MA, USA).

\section{High resolution melting analysis}

For HRM analysis, fluorescent signals were detected at $0.2^{\circ} \mathrm{C}$ intervals, with hold-time for $10 \mathrm{~s}$, between $60-95^{\circ} \mathrm{C}$. Data analysis was performed using PikoReal 2.1 Software (Thermo Fisher Scientific, Walthan, MA, USA).

\section{Sensitivity assays}

For sensitivity assays we tested the performance of the three amplicons with standard strains which produced an overlapped melting profile (see Table 1 for information about the strains).

For amplicon 1, we tested $L$. (L.) donovani, $L$. (L.) mexicana, $L$. $(V$.) braziliensis and $L$. $(V$.$) guyanensis. For ampli-$ con 2 , we tested $L$. (L.) donovani, $L$. (L.) mexicana, $L$. (L.) infantum and $L$. (L.) tropica. For amplicon 3 , we tested $L$. $(L$.) donovani, $L .(L$.) mexicana, $L$. $(V$.) braziliensis and $L$. $(V$.) guyanensis. For all amplicons, a range of parasite DNA from $25 \mathrm{ng}$ to $100 \mathrm{fg}$ was tested, with or without
$25 \mathrm{ng} / \mu \mathrm{l}$ of human DNA. The DNA from standard strains was purified from in vitro cultivated parasites, as described above. Efficiency calculations were made for each amplicon from template ranging from $25 \mathrm{ng}$ to 50 pg (see Additional file 4: Figure S3).

\section{Statistics}

All samples were tested in duplicate in at least three independent experiments. One-way ANOVA was used to calculate statistical difference between the Tm's of paired species for each amplicon. The results are presented as mean differences with $95 \%$ confidence intervals, and statistical significance set to $P<0.05$. The results were analyzed and graphs were produced using GraphPad Prism version 7 (Additional file 5).

\section{Results}

\section{Specificity}

Three amplicons were designed based on available sequences from GenBank or TriTryp database [28], considering that aap3 is present in two copies situated in tandem around $4 \mathrm{~kb}$ apart in most of Leishmania species $[16,47]$. As expected, the BLAST search did not reveal any other hits than for Leishmania spp. As shown in Additional file 1: Figure S1, designed primers generated amplicons of the same size for all the species, presenting differences in nucleotide composition. To predict and to delimit potentially informative polymorphic regions, theoretical melting temperatures were calculated in silico using the OligoCalc tool [29] (data not shown), to guide primer design. In addition, the two copies of the gene were aligned concomitantly for the choice of primers that amplify both copies (data not shown). Although some single nucleotide polymorphism (SNP's) were detected between the two copies of the gene, it did not alter the Tm values. The alignments also allowed the selection of conserved regions for the design of oligonucleotides common to all species, which were used to generate products containing differences in nucleotide composition that were able to distinguish species or groups of species. Furthermore, we analyzed the amplification profile by conventional PCR using the same primers (Additional file 1: Figure S1). We observed specific products of the same size for all species analyzed for each set of primers: 123, 131 and 140 bp for amplicon 1, amplicon 2 and amplicon 3, respectively. No formation of dimers of primers were detected. Control samples were used and no amplification was observed for T. brucei, T. cruzi, E. schaudinni, rat, mouse and human DNA (Additional file 1 and Additional file 2). The analysis of amplicon 3 showed an amplification of $C$. fasciculata, a non-pathogenic and closely related organism to Leishmania spp., but the $\mathrm{Cq}$ and $T m$ values were able to distinguish $C$. fasciculata from all Leishmania spp. tested (Additional file 1: Figure S1 and 
Additional file 2: Figure S2 ). Individual Tm values and normalized melting profiles of 3 amplicons for reference strains are presented in Fig. 1, indicating that these differential profiles distinguished among Leishmania spp. To reliably distinguish different species, we only considered differences in $\mathrm{Tm}$ values exceeding $\pm 0.25{ }^{\circ} \mathrm{C}$, an interval statistically determined as discriminatory (Additional file 5 : Figure S3).
According to Tm and melting curves, amplicon 1 was able to distinguish the species into 3 clusters for subgenus $L$. (Leishmania): the visceral $L$. $(L$.) donovani and L. (L.) infantum, cutaneous strains from Eurasia and Africa $[L$. (L.) major and $L$. (L.) tropica], and cutaneous and mucocutaneous strains from the Americas $L$. (L.) amazonensis, $L$. (L.) mexicana, and strains of subgenus L. (Viannia). Furthermore, it was also able to distinguish

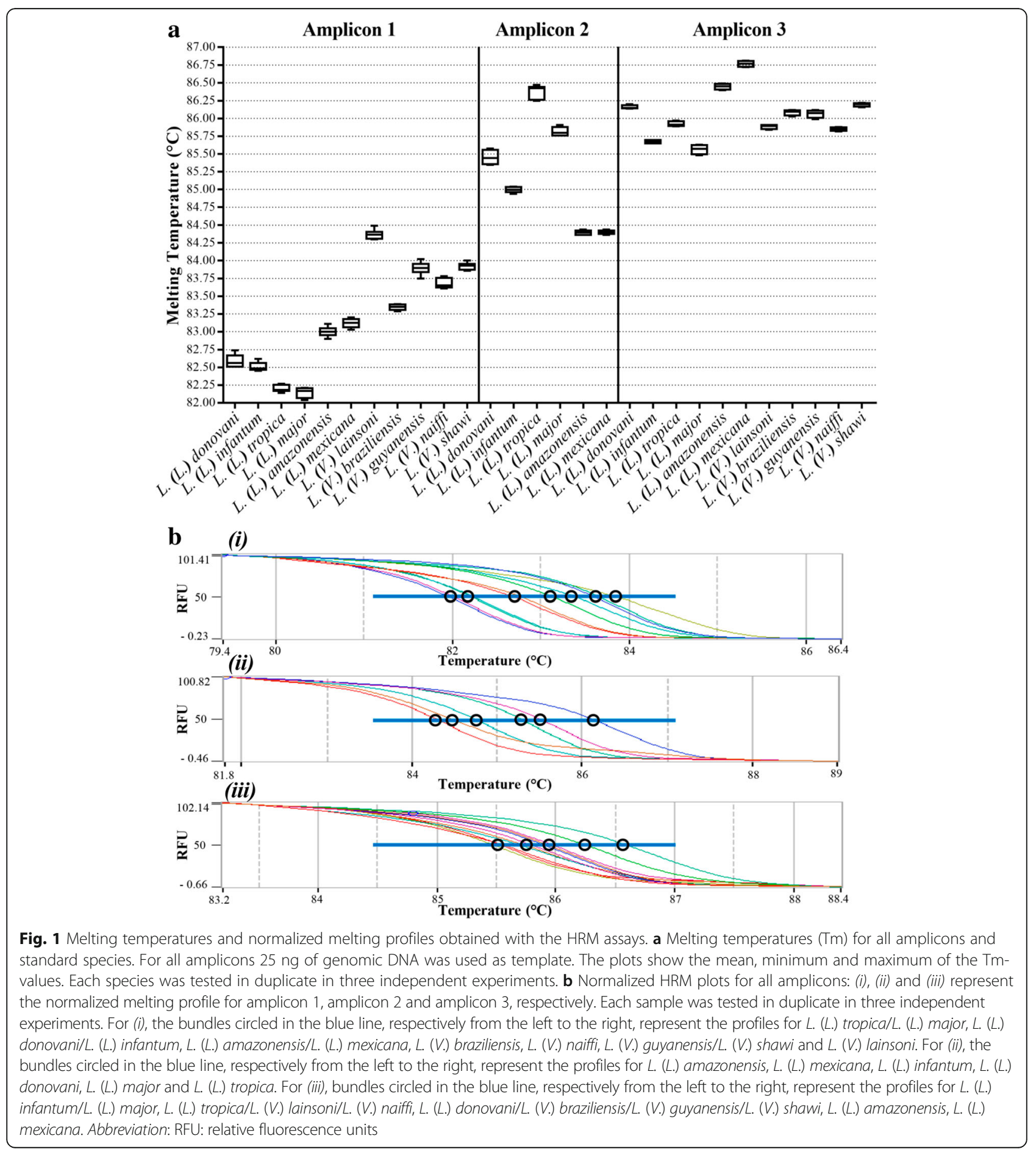


some species of the subgenus L. (Viannia) into 3 clusters: $L$. (V.) lainsoni, $L$. (V.) braziliensis from the other species in that subgenus (Fig. 1).

Amplicon 2 was especially designed for strains of the subgenus L. (Leishmania). No amplification for subgenus L. (Viannia) was observed (Additional file 1: Figure S1). It was able to further distinguish the visceral strains into two different clusters: $L$. (L.) donovani and $L$. (L.) infantum. For the cutaneous species, it was able to distinguish the Eurasian and African cutaneous strains $L$. (L.) major and $L$. (L.) tropica. However, no difference in melting temperature was detected for the American cutaneous strains $L$. (L.) amazonensis and $L$. (L.) mexicana (Fig. 1 ). Therefore, amplicon 3 was designed for this purpose.

Amplicon 3 was, as predicted, able to further distinguish $L$. (L.) amazonensis from $L$. $(L$.) mexicana. Furthermore, it was able to distinguish $L$. (L.) donovani from $L$. ( $L$.) infantum, thereby distinguishing the two species commonly causing VL. It was also able to distinguish the more common causes of CL in Eurasia and Africa: $L$. (L.) major and L. (L.) tropica.

Differences in amplification efficiencies could be observed when the amplification curves from real-time PCR were analyzed. Cq (quantification cycle) data were used as a relative parameter of quantification for the 3 targets when normalized amounts of samples were compared (see Additional file 2: Figure S2). Using $25 \mathrm{ng}$ as template, for all Leishmania species, the amplification reactions produced curves with similar $\mathrm{Cq}$ values for amplicon 1 and 3, indicating a good specificity for all species. For amplicon 2, no amplification of DNA from subgenus $L$. (Viannia) was observed. In addition, high Cq-values for $L$. (L.) amazonensis, $L$. ( $L$.) major and $L$. $(L$.$) mexicana indicate a lower efficiency compared with$ the other L. (Leishmania) species, which also could be used as a parameter of discrimination. For amplicon 1, there was an unspecific amplification of human DNA. However, both the $\mathrm{Cq}$ and $\mathrm{Tm}$ values were distinct.

For all amplicons, additional strains were tested for specificity for all species, except $L$. (L.) infantum, $L$. (L.) amazonensis, $L$. $(V$.) lainsoni and $L$. $(V$.) shawi, as we did not have additional strains for these species available. For amplicon 1 , we found that most strains, except $L$. $(L$.) major and $L$. (V.) naiffi showed a consistent profile for their melting temperatures. For amplicon 2 a relatively large SD was found for $L .(L$.$) donovani (Table 3$ ).

\section{Sensitivity}

To evaluate the role of DNA-concentration, we tested the performance of the three amplicons in strains that presented close melting temperatures, based on the specificity assays. The limit of detection (LOD) was estimated using ten-times serial dilutions from $25 \mathrm{ng}$ to 100 fg of DNA from standard strains purified from in vitro
Table 3 Average melting temperatures and standard deviations (SD) for standard strains and additional strains (see Table 1 for further information on all strains tested)

\begin{tabular}{|c|c|c|c|c|c|c|}
\hline \multirow[t]{2}{*}{ Species } & \multicolumn{2}{|c|}{ Amplicon 1} & \multicolumn{2}{|c|}{ Amplicon 2} & \multicolumn{2}{|c|}{ Amplicon 3} \\
\hline & Average & SD & Average & SD & Average & SD \\
\hline L. (L.) donovani & 82.47 & 0.16 & 85.23 & 0.30 & 86.04 & 0.08 \\
\hline L. (L.) infantum & 82.51 & na & 85.02 & na & 85.67 & na \\
\hline L. (L.) tropica & 82.22 & 0.02 & 86.47 & 0.08 & 85.91 & 0.07 \\
\hline L. (L.) major & 82.29 & 0.24 & 85.90 & 0.11 & 85.57 & 0.04 \\
\hline L. (L.) amazonensis & 83.00 & na & 84.43 & na & 86.45 & na \\
\hline L. (L.) mexicana & 83.12 & 0.00 & 84.46 & 0.05 & 86.88 & 0.09 \\
\hline L. (V.) lainsoni & 84.39 & na & na & na & 85.88 & na \\
\hline L. (V.) braziliensis & 83.44 & 0.18 & na & na & 85.97 & 0.04 \\
\hline L. (V.) guyanensis & 83.83 & 0.15 & na & na & 86.14 & 0.11 \\
\hline L. $(V$.$) naiffi$ & 83.89 & 0.26 & na & na & 86.00 & 0.21 \\
\hline L. $(V$.$) shawi$ & 83.92 & na & na & na & 86.20 & na \\
\hline
\end{tabular}

Abbreviation: na not applicable, where only one strain was tested

cultured parasites. For amplicon 1 the LOD was $100 \mathrm{fg}$ for all species tested except $L$. (L.) mexicana, which had a LOD of $250 \mathrm{fg}$. For amplicon 2 the LOD was $100 \mathrm{fg}$ for all species tested except for $L$. (L.) mexicana, which had a LOD of $50 \mathrm{pg}$. For amplicon 3, the LOD was lower for all species, with $500 \mathrm{fg}$ for $L$. $(L$.$) donovani, L$. (L.) mexicana and $L$. (L.) guyanensis, while for $L$. (V.) braziliensis the LOD was $50 \mathrm{pg}$. The LOD was the same for all amplicons and all species in mixtures of Leishmania and human DNA (data not shown). Considering that the Leishmania genome sizes range between $29 \mathrm{Mb}$ and 33 $\mathrm{Mb}$, varying from 34 to 36 chromosomes [30], the estimated single-cell DNA is approximately $75 \mathrm{fg}$. Thus, we can assume that $100 \mathrm{fg}$ is equivalent to just above one parasite in most of the Leishmania species.

We further tested if the initial amount of DNA would cause a variation in the melting temperature by serially diluting DNA from $25 \mathrm{ng}$ to $100 \mathrm{fg}$ with or without 25 $\mathrm{ng} / \mu \mathrm{l}$ of human DNA. For all three amplicons, the results were the same with or without human DNA. There was a slight variation in melting temperature for all the species, only exceeding $0.2{ }^{\circ} \mathrm{C}$ for $L$. $(V$. $)$ guyanensis in amplicon 1 with human DNA (Table 4). Furthermore, for amplicon 1 the difference in melting temperature between $L$. (L.) mexicana and $L$. $(V$.) braziliensis could potentially cause a confusion between these two species if melting temperatures were considered for this amplicon, the same can be noticed for $L$. $(L$.) donovani, $L$. (L.) braziliensis and L. (L.) guyanensis for amplicon 3 (Fig. 2).

\section{Efficiency}

The reaction efficiency for each amplicon was performed using $25 \mathrm{ng}, 5 \mathrm{ng}$ and $50 \mathrm{pg}$ of DNA from each reference Leishmania species. Efficiency curves, slopes and $R^{2}$ 
Table 4 Effect of initial amount of DNA on melting temperature. Mean and standard deviation (SD) for serial dilutions of DNA from standard strains. DNA concentration ranged from $25 \mathrm{ng}$ to $100 \mathrm{fg}$. Human DNA concentration was kept constant at $25 \mathrm{ng} / \mu \mathrm{l}$

\begin{tabular}{lll}
\hline Strain & $\begin{array}{l}\text { Without human DNA } \\
\text { Mean Tm } \pm \text { SD }\left({ }^{\circ} \mathrm{C}\right)\end{array}$ & $\begin{array}{l}\text { With human DNA } \\
\text { Mean Tm } \pm \text { SD }\left({ }^{\circ} \mathrm{C}\right)\end{array}$ \\
\hline Amplicon 1 & \\
L. (L.) donovani & $82.52 \pm 0.10$ & $82.64 \pm 0.18$ \\
L. (L.) mexicana & $83.16 \pm 0.10$ & $83.23 \pm 0.13$ \\
L. (V.) braziliensis & $83.31 \pm 0.09$ & $83.43 \pm 0.11$ \\
L. (V.) guyanensis & $83.83 \pm 0.08$ & $83.96 \pm 0.34$ \\
Amplicon 2 & & \\
L. (L.) donovani & $85.44 \pm 0.14$ & $85.44 \pm 0.14$ \\
L. (L.) mexicana & $84.40 \pm 0.08$ & $84.56 \pm 0.07$ \\
L. (L.) infantum & $84.92 \pm 0.11$ & $85.17 \pm 0.14$ \\
L. (L.) tropica & $86.33 \pm 0.13$ & $86.73 \pm 0.18$ \\
Amplicon 3 & & $86.15 \pm 0.12$ \\
L. (L.) donovani & $86.17 \pm 0.12$ & $86.91 \pm 0.16$ \\
L. (L.) mexicana & $86.77 \pm 0.16$ & $86.17 \pm 0.15$ \\
L. (V.) braziliensis & $86.00 \pm 0.14$ & $86.36 \pm 0.13$ \\
L. (V.) guyanensis & $85.97 \pm 0.13$ &
\end{tabular}

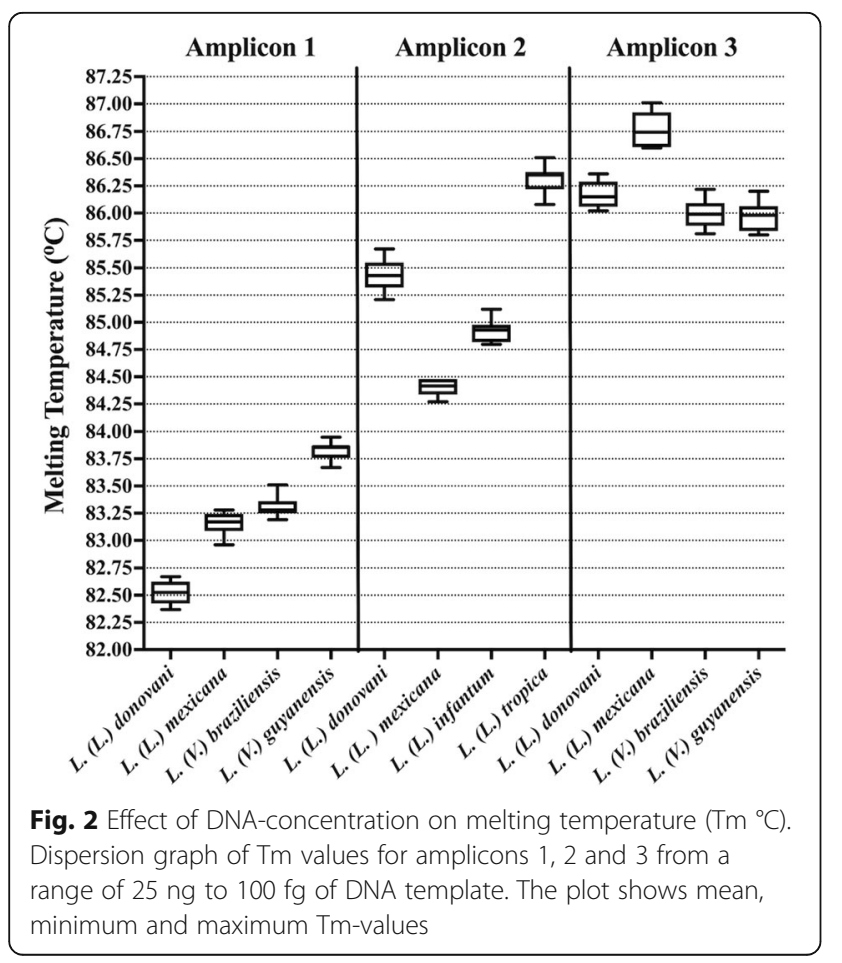

values were calculated (see Additional file 4: Figure S3). The template points produced $\mathrm{Cq}$ values between 20 and 30 , where the PCR reactions produced copies of template at exponential ratios and with $95-105 \%$ of efficiency. The efficiency patterns were similar for all species testes for each amplicon, except for $L$. (L.) mexicana in amplicon 2. The lower efficiency for $L$. (L.) mexicana for amplicon 2 can be explained by the presence of mismatches comparing in primers (see Additional file 3), as can also be observed in the $\mathrm{Cq}$ values showed in Additional file 2: Figure S2.

\section{Validation using naturally and experimentally infected samples}

To validate the aap3-HRM protocol with other samples than reference strain cultures, we applied this protocol in seven biological samples from naturally infected humans, naturally infected cat, naturally infected sand flies, and experimentally infected BALB/c mice (Table 5). The results of amplicon 1 showed a correlation with those obtained with other diagnostic targets; small subunit ribosomal DNA (SSU rDNA) [26], glucose-6-phosphate dehydrogenase $(g 6 p d)$ PCR [31] and heat-shock protein 70 (hsp70) HRM [14]. Although amplicons 2 and 3 were not able to amplify all samples, the positive samples correlated with results obtained with other targets.

\section{Discussion}

Diagnosing Leishmania infection at the species level is important, as it may guide treatment options and followup protocols. Accurate diagnosis is also important in an ecological and epidemiological sense. Unfortunately, in most endemic regions, the leishmaniases are underreported, and the true burden of the disease remains unknown. Techniques that target genomic or mitochondrial DNA by PCR or related techniques are today the most common for detection and identification of Leishmania spp. However, there is no gold standard in methods and targets [8]. Common targets such as kinetoplast DNA (kDNA) and SSU rDNA gene have been used for parasite detection. On the other hand, the g6pd coding region, internal transcribed spacer 1 (ITS1) rRNA, and $h s p 70$ coding region have been more commonly used to identify the parasite at the species level [14, 32-38]. Conventional PCR and real-time PCR often followed by sequencing is increasingly being used for detection and identification of Leishmania spp. [6, 39]. Both methods are relatively laborious and time-consuming and they require PCR product manipulation, increasing the risk of laboratory contamination. Furthermore, the interpretation of the results requires also considerable skill and experience.

HRM is a relatively new real-time PCR coupled technique, with the first papers appearing in 2003 [40, 41]. The technique identifies nucleotide composition polymorphisms 
Table 5 Identification of Leishmania in naturally and experimentally infected samples by HRM analysis targeting the aap3 gene

\begin{tabular}{|c|c|c|c|c|c|}
\hline \multirow{2}{*}{$\begin{array}{l}\text { Sample } \\
\text { source }\end{array}$} & \multicolumn{3}{|l|}{ HRM identification } & \multicolumn{2}{|l|}{ Previous diagnosis } \\
\hline & Amplicon 1 & Amplicon 2 & Amplicon 3 & Diagnostic method & Species identification \\
\hline Human $^{a}$ & L. (L.) amazonensis & negative & negative & SSU rDNA sequencing & L. (L.) amazonensis \\
\hline Human $^{\mathrm{b}}$ & L. (L.) infantum & L. (L.) infantum & L. (L.) infantum & SSU rDNA sequencing & L. (L.) infantum \\
\hline $\mathrm{Cat}^{\mathrm{c}}$ & L. (L.) infantum & L. (L.) infantum & L. (L.) infantum & hsp70 HRM & L. (L.) infantum \\
\hline Mouse $^{d}$ & L. (L.) amazonensis & L. (L.) amazonensis & L. (L.) amazonensis & SSU rDNA sequencing & L. (L) amazonensis \\
\hline Mouse $^{d}$ & L. $(V$.$) braziliensis$ & negative & L. $(V$.$) braziliensis$ & g6pd PCR & L. $(V$.$) braziliensis$ \\
\hline Sand flies ${ }^{e}$ & L. (L.) infantum & L. (L.) infantum & L. (L.) infantum & SSU rDNA sequencing & L. (L.) infantum \\
\hline Sand flies ${ }^{f}$ & Subgenus Viannia & negative & Subgenus Viannia & g6pd PCR & L. $(V$.$) braziliensis$ \\
\hline
\end{tabular}

Note: The aap3 amplicons 1, 2 and 3 of DNA from each sample was submitted to HRM analysis. The result was compared with previous identification performed by SSU rDNA-sequencing [26], hsp70 HRM [14] or g6pd PCR [31]

aHuman paraffin-embedded tissue from Hospital das Clínicas de São Paulo

${ }^{b}$ Human paraffin-embedded tissue from Irmandade da Santa Casa de Misericórdia de São Paulo

Isolated parasites from cat

dExperimentally infected BALB/c mice

${ }^{\mathrm{e}}$ Naturally infected Lutzomyia (Lutzomyia) longipalpis

${ }^{f}$ Naturally infected Lu. (Nyssomyia) whitmani

in real-time PCR products. The HRM methodology presents several attractive features: the whole process is performed in a closed-tube system (avoiding contamination in the laboratory) and it is relatively fast and cheap. The analysis may also be automated. The melting temperature generated depends on a range of factors, where GC-content, sequence, and length of the sequence are central. The melting curves can be objectively differentiated from each other by differences in melting temperature and shape [9].

For Leishmania, there are few studies utilizing HRM for detection and species identification. One of the earliest reports on the usefulness of HRM in differentiation of Leishmania species is by Nicolas et al. [42] who utilized polymorphisms in the coding sequence for minicircle kDNA to differentiate Eurasian and African species, $L$. (L.) major, $L$. (L.) donovani and $L$. (L.) tropica, and $L$. (L.) infantum. Later, Talmi-Frank et al. [43] utilized the ITS1 rRNA region to identify, distinguish and quantify Eurasian and African species, L. (L.) infantum/L. (L.) donovani, $L$. (L.) aethiopica, $L$. (L.) tropica and $L$. (L.) major. Both Nicolas et al. [42] and Talmi-Frank et al. [43] only targeted Eurasian and African species, making the approaches valuable in these endemic areas, although of limited value in other endemic areas and in a non-endemic settings where species from all endemic regions could be expected. Pita-Pereira et al. [33] also utilized the minicircle kDNA to discriminate among strains from the subgenus $L$. (Viannia) and $L$. (L.) infantum and $L$. (L.) amazonensis, making it an attractive methodology in an American setting to differentiate strains commonly causing cutaneous, mucocutaneous and visceral manifestations. Ceccarelli et al. [44] also demonstrated an ability to differentiate species of the subgenus $L$. (Leishmania) from L. (Viannia). HRM has also been used for detection of Leishmania in sand flies, where Aghaei et al. [45] used ITS1 to identify $L$. (L.) tropica in sand flies. Kuang et al. [46] utilized the lack gene to differentiate five Eurasian and African species from one American species, $L$. $(V$.) braziliensis. Although they showed its usefulness in clinical samples, the relatively few Leishmania species tested made it difficult to conclude if this target could be useful in other endemic areas. With increasing travel to several endemic regions, the correct diagnosis and species identification for the leishmaniases is of paramount interest. Hernandez et al. [36] utilized the ITS1 and $h s p 70$ to differentiate $L$. (L.) mexicana, $L$. (L.) infantum, $L .(L$.$) amazonensis, L .(V$.$) panamensis, L .(V$.) guyanensis and $L .(V$.$) braziliensis. Although they found$ some ambiguities in species identification between the targets, they showed that ITS1 and $h s p 70$ had potential as diagnostic targets utilizing HRM. Zampieri et al. [14] showed, in a recent paper, that HRM targeting several polymorphic sites on the $h s p 70$ coding region could successfully be used to differentiate several Eurasian, African and American species. This makes it the most attractive target reported so far, especially useful in a non-endemic setting where the patient could have travelled to several endemic areas.

In this work, we describe a method for rapid detection and discrimination of most Leishmania species. We used the aap3 coding sequence as target. Leishmania aap3 coding sequences available in the GenBank and TriTryp databases were aligned to search for relatively conserved regions but present polymorphisms that enable the identification of different species. For this, the available coding sequences of some species from the subgenus $L$. (Leishmania) and L. (Viannia) were analyzed in silico. The coding sequence for aap3 is present in two copies and organized in tandem in most of the Leishmania spp. genomes. For $L$. $(L$.) donovani and $L$. $(L$.) amazonensis a $98 \%$ identity has been described between the copies of coding regions and a 93\% identity between these two 
species [16, 47]. In addition, aap3 appeared conserved among other Leishmania species [17]. Although some species present only one aap3 gene copy, for example $L$. $(V$.$) braziliensis, this could be due the misannotation in$ the genome database. Considering these observations, the polymorphisms found between the two copies of the coding region did not affect the Tm analysis.

Amplicon 1 was able to discriminate the two strains causing visceral leishmaniasis from strains causing American cutaneous leishmaniasis. Furthermore, it was able to differentiate the American cutaneous strains and several of the strains of the subgenus $L$. (Viannia). The inability to differentiate the two visceral strains $L$. $(L$.) donovani from $L$. (L.) infantum was compensated with these strains having distinct Tm's in amplicon 2 and amplicon 3. The same situation is true for the Eurasian cutaneous species: $L$. (L.) major and $L$. (L.) tropica were indistinguishable in amplicon 1, but had distinct Tm's in amplicons 2 and 3 . Leishmania (L.) amazonensis and $L$. (L.) mexicana are phylogenetically closely related species [48], and the difficulty in differentiating these species for diagnostic purposes has been described elsewhere [14]. Both amplicon 1 and 2 showed similar profiles for these two species, while amplicon 3 was able to distinguish them. Amplicon 2 was specifically designed for the subgenus $L$. (Leishmania), and there was no amplification of $L$. (Viannia) spp. High Cq values, observed for $L$. (L.) amazonensis, $L$. (L.) mexicana and $L$. (L.) major for amplicon 2, could be explained by primer-mismatches. Some caution should be taken when analyzing the result in a diagnostic setting, especially if negative. However, taken together the results from all amplicons can be considered to strengthen the diagnosis.

Diagnosing leishmaniasis relies on patient and travel history, clinical information (symptoms and clinical findings), and results from laboratory tests. Thus, for example, the inability of amplicon 1 to reliably distinguish $L$. (L.) donovani from $L$. (L.) infantum, in a patient with suspected visceral leishmaniasis is of little importance in a clinical setting, as the patient would receive the same treatment and follow-up regime regardless. But in an epidemiological setting, it is of importance to distinguish the species from each other to generate reliable data.

In general, multi-copy genes can be expected to yield a higher sensitivity in molecular diagnostics. The assays developed by Talmi-Frank et al. [43], Hernandez et al. [36] and Zampieri et al. [14], all report a limit of detection (LOD) of less than one parasite. Despite that aap3 only comes in two copies we report a relatively good sensitivity with a LOD of $100 \mathrm{fg}$ for amplicon 1 (just above 1 parasite) to $500 \mathrm{fg}$ for amplicon 3 (approximately 5 parasites). This could probably be improved with a pre-amplification step.

Some HRM assays have found little evidence that DNA concentration of the initial template influences the Tm $[36,46]$. However, the initial amount of DNA for

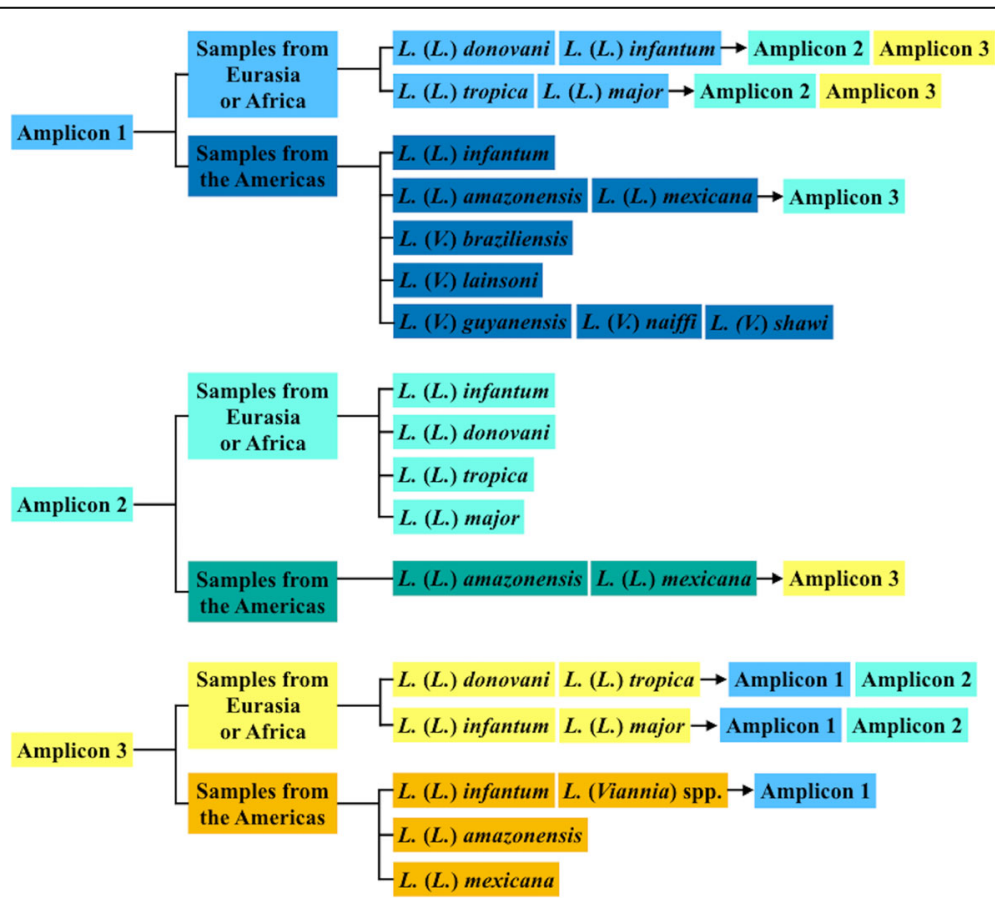

Fig. 3 Proposed strategy for Leishmania species identification. Diagram of a proposed strategy using aap3-HRM for species identification. For VL patients from the Americas, amplicon 1 would suffice, while for VL patients from Eurasia and Africa we suggest the use of either amplicon 2 alone or amplicon 1 and 2. For patients with $\mathrm{CL}$ and $\mathrm{MCL}$ form the Americas, we suggest the use of both amplicon 1 and 3 
this assay influenced the Tm for some of the species. This is in concordance with the findings of Zampieri et al. [14], who also found that initial DNA applied to the assay did affect the Tm for several strains. For the amplicons investigated in our study, this could lead to a misidentification for $L$. (L.) mexicana and $L$. (L.) braziliensis for amplicon 1. However, it should be noted that we propose the use of the three amplicons in the identification to strengthen the diagnostic validity and avoid species misidentification. A strategy for Leishmania species identification in patients with suspected leishmaniases is proposed in Fig. 3.

The validation of the target and the technique with clinical and experimentally infected samples from human, cat, mice and sand flies indicated a good correlation with other diagnostic targets and techniques. Not all amplicons were able to yield a result for all the samples, as could be expected due to differences in specificity and sensitivity of the primers, where amplicon 2 was not produced for any species belonging to the subgenus L. (Viannia). Despite the limited number of samples tested, the data indicate the potential of the aap3-HRM method to identify Leishmania species.

\section{Conclusions}

Overall, the aap3 coding sequence can be a promising target since it is specific and conserved for Leishmania spp. The design of the aap3-HRM protocol described is a relatively rapid, simple, sensitive and specific method to identify and distinguish several Leishmania spp. There is no need for sequencing or gel fractionation to analyze a PCR-product, minimizing the laboratory contamination as all the reactions are performed within a closed tube. The method can be automated, dispensing a trained technician to analyze the results. It also has the potential to quantify parasites present in samples, as it is a real-time PCR technique, able to detect a small number of parasites. In conclusion, the protocol described may offer a relatively low-cost and reliable method for detection and identification of Leishmania in biological and clinical samples.

\section{Additional files}

Additional file 1: Figure S1. Agarose gel electrophoresis of $\mathrm{PCR}$ products. Reactions were performed using TopTaq Master Mix (Qiagen, Hilden, Germany) in a final volume of $25 \mu \mathrm{l}$ with $200 \mathrm{nM}$ of each primer and 25 ng of genomic DNA as a template. The PCR product was applied to a $3 \%$ agarose gel and stained with ethidium bromide. Conventional PCR products for standard strains and controls: A, amplicon 1 (expected 123 bp); B, amplicon 2 (expected 131 bp); C, amplicon 3 (expected 140 bp). (DOCX $8881 \mathrm{~kb})$

Additional file 2: Figure S2. Specificity using $\mathrm{Cq}$ values as parameter. Representative graph of Cq values obtained with HRM assays. The same amount of genomic DNA from all species was used as template to evaluate amplification efficiency. The samples used as negative controls are marked in red. Products generated in late Cq's (>30) were evaluated in the PikoReal software and revealed that Tm's and melting profiles were different than for Leishmania. The fluorescence generated for these samples was due to unspecific amplification or noise. (DOCX $152 \mathrm{~kb}$ )

Additional file 3: Alignment of nucleotide sequences of aap3 coding regions and primer localization. The underlined sequences indicate the position of the primers used and the grey boxes represent the variable regions found among the Leishmania strains based on in silico analysis. The numbers at the top of each amplicon are based on the position of the nucleotides in relation to the whole coding sequence in $L$. (L.) amazonensis. (DOCX $21 \mathrm{~kb}$ )

Additional file 4: Figure S3. Efficiency curves for all amplicons. Efficiency curves, slopes and R2 were calculated from four species for each amplicon using $25 \mathrm{ng}, 5 \mathrm{ng}$ and $5 \mathrm{pg}$ of DNA from each Leishmania species. For amplicon 1 and 3 two species of L. (Leishmania) and two of subgenus $L$. (Viannia) were selected. Amplicon 2 only amplified L. (Leishmania), and strains from this subgenus were therefore selected. (DOCX $948 \mathrm{~kb}$ )

Additional file 5: Statistical analysis of melting temperatures (Tm's) for all amplicons. (XLSX $16 \mathrm{~kb})$

\section{Abbreviations}

aap3: Amino acid permease 3; BLAST: Basic local alignment search tool; CL: Cutaneous leishmanisis; Cq: Quantification cycle; DAT: Direct agglutination test; DNA: Deoxyribonucleic acid; g6pd: Glucose-6-phosphate dehydrogenase; HRM: High resolution melting analysis; hsp70: Heat-shock protein 70; ITS1: Internal transcribed spacer 1; kDNA: Kinetoplast DNA; LOD: Limit of detection; PCR: Polymerase chain reaction; SD: Standard deviation; SSU rDNA: Small subunit ribosomal DNA; VL: Visceral leishmaniasis; WHO: World Health Organization

\section{Acknowledgments}

Not applicable.

\section{Funding}

This work was supported by grants from the University of Bergen, Norwegian Centre for International Cooperation in Education (SIU) and Coordenação de Aperfeiçoamento de Pessoal de Nível Superior (CAPES) - collaboration project CAPES-SIU-2015/10002, and Fundação de Amparo a Pesquisa do Estado de São Paulo (FAPESP). None of the funding bodies had any role in the design of the study, data collection, analysis or writing of the manuscript.

\section{Availability of data and materials}

The data supporting the conclusions of this article are included within the article and its additional files. All important datasets are available upon a request.

\section{Authors' contributions}

KEM and RAZ conceptualized, designed, conducted the experiments, analyzed the results and wrote the manuscript. LMFW and AHN conceptualized, contributed to the design of the project, acquired the funding, analyzed the results and reviewed the manuscript. JIA and SMM contributed to the design of the project, analyzed the results and reviewed the manuscript. All authors read and approved the final manuscript.

\section{Ethics approval and consent to participate}

For the human paraffin-embedded samples from patients from Hospital das Clínicas da Universidade de São Paulo or Hospital da Irmandade da Santa Casa de Misericórdia, ethical approval was obtained from the Committee of Ethics of Irmandade de Santa Casa de Misericórdia de São Paulo (61469916.6.1001.0068). For the samples from experimentally infected mice, ethical approval was obtained from Instituto de Biociências - USP, protocol 145 (20/10/2011) Committee of Ethics, USP). The DNA samples used as non-infected controls were obtained from the DNA repository of the Laboratory of Trypanosomatidae at Physiology - IB-USP, Brazil. The ethical approval for these samples were obtained in projects previously approved.

Consent for publication

Not applicable.

\section{Competing interests}

The authors declare that they have no competing interests. 


\section{Publisher's Note}

Springer Nature remains neutral with regard to jurisdictional claims in published maps and institutional affiliations.

\section{Author details}

'Department of Clinical Science, Faculty of Medicine, University of Bergen, Postboks 7804, 5020 Bergen, Norway. ${ }^{2}$ Department of Physiology, Institute of Biosciences, University of São Paulo, Rua do Matão Travessa 14 no. 101, São Paulo, SP 05508900, Brazil.

\section{Received: 15 February 2018 Accepted: 3 July 2018}

\section{Published online: 16 July 2018}

\section{References}

1. Pace D. Leishmaniasis. J Infect. 2014;69:10-8

2. World Health Organization. Neglected Tropical Diseases. http://www.who. int/neglected_diseases/diseases/en/. Accessed 6 Jan 2018.

3. Alvar J, Vélez ID, Bern C, Herrero M, Desjeux P, Cano J, et al. Leishmaniasis worldwide and global estimates of its incidence. PLoS One. 2012;7:e35671.

4. Centers for Disease Control and Prevention. Resource for health professionals: parasites - leishmaniasis. https://www.cdc.gov/parasites/ leishmaniasis/health_professionals/index.html. Accessed 6 Jan 2018.

5. Lainson R, Shaw JJ. Evolution, classification and geographical distribution. In: Peters W, Killick-Kendrick R, editors. The Leishmaniases in Biology and Medicine. London: Academic Press Inc.; 1987. p. 1-120.

6. Aronson N, Herwaldt BL, Libman M, Pearson R, Lopez-Velez R, Weina P, et al Diagnosis and treatment of leishmaniasis: clinical practice guidelines by the Infectious Diseases Society of America (IDSA) and the American Society of Tropical Medicine and Hygiene (ASTMH). Am J Trop Med Hyg. 2017;96:24-45.

7. Cunningham J, Hasker E, Das P, El Safi S, Goto H, Mondal D, et al. A Global comparative evaluation of commercial immunochromatographic rapid diagnostic tests for visceral leishmaniasis. Clin Infect Dis. 2012;55:1312-9.

8. Akhoundi M, Downing T, Votýpka J, Kuhls K, Lukeš J, Cannet A, et al. Leishmania infections: molecular targets and diagnosis. Mol Aspects Med. 2017:57:1-29.

9. Tong SYC, Giffard PM. Microbiological applications of high-resolution melting analysis. J Clin Microbiol. 2012;50:3418-21.

10. Pangasa A, Jex AR, Campbell BE, Bott NJ, Whipp M, Hogg G, et al. High resolution melting-curve (HRM) analysis for the diagnosis of cryptosporidiosis in humans. Mol Cell Probes. 2009;23:10-5.

11. Chua KH, Lim SC, Ng CC, Lee PC, Lim YAL, Lau TP, et al. Development of high resolution melting analysis for the diagnosis of human malaria. Sci Rep. 2015:5:15671.

12. Odell ID, Cloud JL, Seipp M, Wittwer CT. Rapid species identification within the Mycobacterium chelonae-abscessus group by high-resolution melting analysis of hsp65 PCR products. Am J Clin Pathol. 2005;123:96-101.

13. Merchant-Patel S, Blackall PJ, Templeton J, Price EP, Tong SYC, Huygens F, et al. Campylobacter jejuni and Campylobacter coli genotyping by high-resolution melting analysis of a flaA fragment. Appl Environ Microbiol. 2010;76:493-9.

14. Zampieri RA, Laranjeira-Silva MF, Muxel SM, de Lima ACS, Shaw JJ, FloeterWinter LM. High resolution melting analysis targeting hsp70 as a fast and efficient method for the discrimination of Leishmania species. PLoS Negl Trop Dis. 2016;10:e0004485

15. Muxel SM, Aoki Il, Fernandes JCR, Laranjeira-Silva MF, Zampieri RA, Acuña SM, et al. Arginine and polyamines fate in Leishmania infection. Front Microbiol. 2018:8:2682.

16. Shaked-Mishan P, Suter-Grotemeyer M, Yoel-Almagor T, Holland N, Zilberstein D, Rentsch D. A novel high-affinity arginine transporter from the human parasitic protozoan Leishmania donovani. Mol Microbiol. 2006;60:30-8.

17. Aoki Jl, Muxel SM, Zampieri RA, Acuña SM, Fernandes JCR, Vanderlinde RH et al. L-arginine availability and arginase activity: characterization of amino acid permease 3 in Leishmania amazonensis. PLoS Negl Trop Dis. 2017:11:e0006025.

18. Zilberstein D. Transport of nutrients and ions across membranes of trypanosomatid parasites. Adv Parasitol. 1993;32:261-91.

19. Zilberstein D, Gepstein A. Regulation of I-proline transport in Leishmania donovani by extracellular pH. Mol Biochem Parasitol. 1993;61:197-205.

20. Akerman M, Shaked-Mishan P, Mazareb S, Volpin H, Zilberstein D. Novel motifs in amino acid permease genes from Leishmania. Biochem Biophys Res Commun. 2004;325:353-66
21. da Silva MFL, Zampieri RA, Muxel SM, Beverley SM, Floeter-Winter LM. Leishmania amazonensis arginase compartmentalization in the glycosome is important for parasite infectivity. PLoS One. 2012;7:e34022.

22. Roberts SC, Tancer MJ, Polinsky MR, Gibson KM, Heby O, Ullman B. Arginase plays a pivotal role in polyamine precursor metabolism in Leishmania. Characterization of gene deletion mutants. J Biol Chem. 2004;279:23668-78.

23. Reguera RM, Balaña-Fouce R, Showalter M, Hickerson S, Beverley SM. Leishmania major lacking arginase (ARG) are auxotrophic for polyamines but retain infectivity to susceptible BALB/c mice. Mol Biochem Parasitol. 2009;165:48-56

24. Tellevik MG, Muller KE, Løkken KR, Nerland AH. Detection of a broad range of Leishmania species and determination of parasite load of infected mouse by real-time PCR targeting the arginine permease gene AAP3. Acta Trop. 2014;137:99-104.

25. Control of the leishmaniases. Report of a WHO Expert Committee. World Health Organ Tech Rep Ser. 1990;793:1-158

26. Uliana SR, Nelson K, Beverley SM, Camargo EP, Floeter-Winter LM. Discrimination amongst Leishmania by polymerase chain reaction and hybridization with small subunit ribosomal DNA derived oligonucleotides. J Eukaryot Microbiol. 1994;41:324-30.

27. Uliana SR, Affonso MH, Camargo EP, Floeter-Winter LM. Leishmania: genus identification based on a specific sequence of the 185 ribosomal RNA sequence. Exp Parasitol. 1991;72:157-63.

28. Aslett M, Aurrecoechea C, Berriman M, Brestelli J, Brunk BP, Carrington M, et al. TriTrypDB: a functional genomic resource for the Trypanosomatidae. Nucleic Acids Res. 2010;38:457-62.

29. Kibbe WA. OligoCalc: an online oligonucleotide properties calculator. Nucleic Acids Res. 2007:35:43-6.

30. Real F, Vidal RO, Carazzolle MF, Mondego JMC, Costa GGL, Herai RH, et al. The genome sequence of Leishmania (Leishmania) amazonensis: functional annotation and extended analysis of gene models. DNA Res. 2013;20:567-81.

31. Castilho TM, Shaw JJ, Floeter-Winter LM. New PCR assay using glucose-6phosphate dehydrogenase for identification of Leishmania species. J Clin Microbiol. 2003:41:540-6.

32. Van der Auwera G, Maes I, De Doncker S, Ravel C, Cnops L, Van Esbroeck M, et al. Heat-shock protein 70 gene sequencing for Leishmania species typing in European tropical infectious disease clinics. Eurosurveillance. 2013;18:20543.

33. Pita-Pereira D, Lins R, Oliveira MP, Lima RB, Pereira BA, Moreira OC, et al. SYBR Green-based Real-Time PCR targeting kinetoplast DNA can be used to discriminate between the main etiologic agents of Brazilian cutaneous and visceral leishmaniases. Parasit Vectors. 2012;5:15

34. Tsukayama P, Lucas C, Bacon DJ. Typing of four genetic loci discriminates among closely related species of New World Leishmania. Int J Parasitol. 2009;39:355-62

35. Mary C, Faraut F, Lascombe L, Dumon H. Quantification of Leishmania infantum DNA by a real-time PCR assay with high sensitivity. J Clin Microbiol. 2004;42:5249-55.

36. Hernández C, Alvarez C, González C, Ayala MS, León CM, Ramírez JD. Identification of six New World Leishmania species through the implementation of a high-resolution melting (HRM) genotyping assay. Parasit Vectors. 2014:7:501.

37. da Graça GC, Volpini AC, Romero GAS, de Oliveira Neto MP, Hueb M, Porrozzi R, et al. Development and validation of PCR-based assays for diagnosis of American cutaneous leishmaniasis and identificatio nof the parasite species. Mem Inst Oswaldo Cruz. 2012:107:664-74.

38. Jara M, Adaui V, Valencia BM, Martinez D, Alba M, Castrillon C, et al. Realtime PCR assay for detection and quantification of Leishmania (Viannia) organisms in skin and mucosal lesions: exploratory study of parasite load and clinical parameters. J Clin Microbiol. 2013;51:1826-33.

39. Van der Auwera G, Bart A, Chicharro C, Cortes S, Davidsson L, Di Muccio T, et al. Comparison of Leishmania typing results obtained from 16 European clinical laboratories in 2014. Eurosurveillance. 2016:21:49.

40. Gundry CN. Amplicon melting analysis with labeled primers: a closed-tube method for differentiating homozygotes and heterozygotes. Clin Chem. 2003;49:396-406.

41. Wittwer CT. High-resolution genotyping by amplicon melting analysis using LCGreen. Clin Chem. 2003;49:853-60.

42. Nicolas L, Milon G, Prina E. Rapid differentiation of Old World Leishmania species by LightCycler polymerase chain reaction and melting curve analysis. J Microbiol Methods. 2002;51:295-9.

43. Talmi-Frank D, Nasereddin A, Schnur LF, Schönian G, Töz SÖ, Jaffe CL, et al. Detection and identification of Old World Leishmania by high resolution melt analysis. PLoS Negl Trop Dis. 2010;4:e581. 
44. Ceccarelli M, Galluzzi L, Migliazzo A, Magnani M. Detection and characterization of Leishmania (Leishmania) and Leishmania (Viannia) by SYBR Green-based realtime PCR and high resolution melt analysis targeting kinetoplast minicircle DNA. PLoS One. 2014:9:e88845.

45. Aghaei AA, Rassi Y, Sharifi I, Vatandoost H, Mollaie H, Oshaghi M, et al. First report on natural Leishmania infection of Phlebotomus sergenti due Leishmania tropica by high resolution melting curve method in south-eastern Iran. Asian Pac J Trop Med. 2014;7:93-6.

46. Kuang Z, Zhang C, Pang H, Ma Y. A rapid high-resolution melting method for differentiation of Leishmania species targeting lack gene. Acta Trop. 2018:178:103-6.

47. Castilho-Martins EA, Laranjeira da Silva MF, dos Santos MG, Muxel SM, Floeter-Winter LM. Axenic Leishmania amazonensis promastigotes sense both the external and internal arginine pool distinctly regulating the two transporter-coding genes. PLoS One. 2011;6:e27818.

48. Tschoeke DA, Nunes GL, Jardim R, Lima J, Dumaresq ASR, Gomes MR, et al. The Comparative genomics and phylogenomics of Leishmania amazonensis parasite. Evol Bioinform Online. 2014;10:131-3.

Ready to submit your research? Choose BMC and benefit from:

- fast, convenient online submission

- thorough peer review by experienced researchers in your field

- rapid publication on acceptance

- support for research data, including large and complex data types

- gold Open Access which fosters wider collaboration and increased citations

- maximum visibility for your research: over $100 \mathrm{M}$ website views per year

At BMC, research is always in progress.

Learn more biomedcentral.com/submissions 